\title{
Effects of decoherence on the radiative and squeezing properties in a coherently driven trapped two-level atom
}

\author{
Sintayehu Tesfa \\ Physics Department, Addis Ababa University, P. O. Box 1176, Addis Ababa, Ethiopid*
}

(Dated: January 15, 2019)

\begin{abstract}
Analysis of the effects of decoherence on the radiative and squeezing properties of a coherently driven two-level atom trapped in a resonant cavity applying the corresponding master equation is presented. The atomic dynamics as well as the squeezing and statistical properties of the emitted radiation are investigated. It is found that the atom stays in the lower energy level more often at steady state irrespective of the strength of the coherent radiation and thermal fluctuations entering the cavity. Moreover, a strong external coherent radiation results the splitting of the line of the emission spectrum, whereas the decoherence broadens the width and significantly decreases the height. It is also found that the emitted radiation exhibits photon anti-bunching, super-Poissonian photon statistics and squeezing, despite the presence of the decoherence which is expected to destroy the quantum features.
\end{abstract}

Keywords: two-level atom, atomic dynamics, quadrature squeezing, emission spectrum, decoherence

\section{INTRODUCTION}

Interaction of a single two-level atom with a radiation has received a great deal of interest in recent years [1, 2, 3. 4, 5, 6, 7, 8, 9, 10, 11, 12, 13, 14, 15, 16, 17, 18, 19]. In the absence of the external light, spontaneous emission of an excited two-level atom results due to the fluctuations in a continuum of vacuum modes that play a role of a reservoir. However, when the vacuum modes are replaced with, let us say, a squeezed vacuum reservoir, the radiative properties of the atom are significantly modified [1, 2, 3, 4, 5, ㅎ, ㄱ, ]. In this respect, the resonance fluorescence and absorption spectra of a driven two-level atom coupled to a squeezed vacuum reservoir have been analyzed by various authors [1, 2]. It is, in general, found that the rate of emission is greater than the rate of absorption, in which the squeezed input inhibits absorption somehow and broadens the spectrum with decreasing height. Moreover, whenever the atom is driven on resonance by a strong external monochromatic laser beam, the structure of the atomic energy level changes dramatically, that is, the atomic dynamics and properties of the emitted radiation would be appreciably altered. It is known for long that the resonance fluorescence spectrum splits into Mollow triplets in the strong driving regime [9]. It is also a well-established fact that the radiative properties of the atom and squeezing properties of the emitted radiation considerably depend on the amplitude of the driving radiation.

It can be learned from earlier works that the successively emitted photons from the two-level atom in the cavity are correlated due to the atomic coherence induced by the driving mechanism, whereby the emitted radiation is found to exhibit nonclassical features. For instance, D'Souza et al. [10] analyzed the quantum nature of the

*Electronic address: sint tesfa@yahoo.com light emitted by the two-level atom coupled to a squeezed vacuum reservoir in the strong driving limit with the aid of atomic-dressed state earlier. Based on the phase sensitivity of the Mandel's response function, they claimed that the emitted light exhibits squeezing. Most recently, the squeezing properties of the radiation emitted by a coherently driven two-level atom coupled to a broadband squeezed vacuum reservoir is studied using the variance of the atomic-dipole operator in the normal ordering. Successively emitted radiation turns out to be in squeezed state even in the absence of the squeezed input for certain values of the amplitude of the external radiation. In addition, although it was predicted earlier that the emitted radiation exhibits sub and super-Poissonian photon statistics, from the curve of the Mandel's response function [10], recent analysis based on the two-time secondorder correlation function shows that it exhibits superPoissonian photon statistics for larger delayed time [1]. It is also found that the two-time second-order correlation function oscillates with the delayed time, where the frequency of its oscillation increases with the amplitude of the driving radiation, whereas its height decays fast with the squeeze parameter.

In the actual experimental setting, the two-level atom in the cavity is unavoidably coupled to the fluctuations in the surrounding environment via the walls of the cavity. In general, the phenomenon in which the quantum system losses its nonclassical features due to its interaction with the environment is defined as decoherence. It is not difficult to realize, therefore, that decoherence is basically related to unbiased noise fluctuations in the modes of the environment that able to interact with the system. Though various ways of including the effects of decoherence are possible [20], its contribution can be readily modeled as thermal fluctuations of the walls of the cavity that can be taken usually as thermal reservoir. It is a well-known fact that a squeezed vacuum reservoir introduces a biased noise fluctuations to the system, as a result it induces additional coherence, whereas 
the thermal reservoir, on the other hand, adds decoherence into the system. In view of the contribution of the squeezed vacuum reservoir towards the nonclassical features of the emitted radiation that have been reported, it appears natural to ask how the radiative properties of the atom as well as the squeezing and statistical properties of the emitted radiation could possibly be modified by decoherence due to the presumed thermal heating entering the cavity via the vibration of the walls of the container? On the basis of the properties of unbiased noise fluctuations associated with the thermal heating, it seams reasonable to expect that the quantum features of the radiation would be degraded by the decoherence. The main task of this work is, thereupon, devoted to investigate this basic issue. Earlier, effects of the thermal light as incoherent relaxation on the collapse and revival as well as the photon anti-bunching have been considered by Puri and Agarwal [21]. They found that the oscillations of the collapse and revival become more irregular with the intensity of the thermal radiation and the thermal light characteristically destroys the photon anti-bunching phenomenon.

In this communication, the effects of decoherence on a radiative, squeezing and statistical properties of a coherently driven two-level atom would be analyzed. It is a common knowledge that the effects of squeezed input are related to the amplitude and phase fluctuations of the reservoir modes. Nonetheless, which of these two would be predominant one of the issues in this work. To achieve this goal, the squeezed input is replaced with an unbiased thermal fluctuations whose mean phase fluctuations are readily averaged out to be zero. In accordance to this, throughout, the results previously obtained for squeezed input elsewhere [1] are compared with the effects of the thermal fluctuations so that which of the fluctuations in the reservoir modes would actually be essential in bringing about a significant modification in a radiative and squeezing properties ie evident. Though methods from the stochastic simulation of the Bloch equations in secular approximation [11, 12] to diagonalizing the coefficient matrix [6, [7, 10] have been used in previous contributions, the differential equations associated with the expectation values of the atomic and energy operators following from the master equation would be simultaneously solved in view of the procedure recently applied. It is believed that this approach helps in overcoming the inevitable limitations corresponding to the approximations and computer simulation frequently employed. Usually the effects of the external coherent radiation either in a weak or strong driving limit have been studied, but in here an arbitrary amplitude of the driving radiation is taken. In particular, the population inversion, probability for the atom to be in the upper energy level, emission spectrum, twotime second-order correlation function and quadrature variance for the cavity radiation in terms of the atomic polarization would be calculated.

\section{ATOMIC DYNAMICS}

It is a common knowledge that driving a two-level atom on resonance by a coherent light amounts to pumping the two-level atom continuously by an external laser beam whose frequency matches with the atomic transition frequency. Treating the driving radiation classically, the Hamiltonian describing the interaction of two-level atom with a radiation in the rotating-wave and electricdipole approximations in the interaction picture can be expressed as

$$
\hat{H}=i \frac{\Omega}{2}\left(\hat{\sigma}_{+}-\hat{\sigma}_{-}\right)
$$

where $\Omega$ is the positive-real constant proportional to the amplitude of the external coherent radiation, $\hat{\sigma}_{+}\left(\hat{\sigma}_{-}\right)$ is the creation (annihilation) atomic operator defined as $\hat{\sigma}_{+}=|a\rangle\langle b|$ and $\hat{\sigma}_{-}=|b\rangle\langle a|$ in which $|a\rangle$ and $|b\rangle$ represent the upper and lower atomic energy levels. It is a well known fact that the master equation of a two-level atom coupled to a thermal reservoir can be derived applying the Born-Markov approximation. Hence following the standard procedure [22], it is possible to verify for a twolevel atom driven on resonance by a coherent light and coupled to a thermal reservoir that

$$
\begin{aligned}
\frac{d \hat{\rho}}{d t} & =\frac{\Omega}{2}\left(\hat{\sigma}_{+} \hat{\rho}-\hat{\rho} \hat{\sigma}_{+}-\hat{\sigma}_{-} \hat{\rho}-\hat{\rho} \hat{\sigma}_{-}\right) \\
& +\frac{\gamma(\bar{n}+1)}{2}\left[2 \hat{\sigma}_{-} \hat{\rho} \hat{\sigma}_{+}-\hat{\sigma}_{+} \hat{\sigma}_{-} \hat{\rho}-\hat{\rho} \hat{\sigma}_{+} \hat{\sigma}_{-}\right] \\
& +\frac{\gamma \bar{n}}{2}\left[2 \hat{\sigma}_{+} \hat{\rho} \hat{\sigma}_{-}-\hat{\sigma}_{-} \hat{\sigma}_{+} \hat{\rho}-\hat{\rho} \hat{\sigma}_{-} \hat{\sigma}_{+}\right]
\end{aligned}
$$

where $\gamma$ is the atomic damping constant and $\bar{n}$ is the mean photon number corresponding to the reservoir modes, which is the measure of the intensity of the unbiased noise fluctuations of the broadband environment modes.

Making use of the master equation (2), the time evolution of the expectation values of the atomic creation, annihilation and energy operators can be obtained,

$$
\begin{array}{r}
\frac{d}{d t}\left\langle\hat{\sigma}_{-}(t)\right\rangle=-\frac{\gamma}{2}(2 \bar{n}+1)\left\langle\hat{\sigma}_{-}(t)\right\rangle-\frac{\Omega}{2}\left\langle\hat{\sigma}_{z}(t)\right\rangle, \\
\frac{d}{d t}\left\langle\hat{\sigma}_{+}(t)\right\rangle=-\frac{\gamma}{2}(2 \bar{n}+1)\left\langle\hat{\sigma}_{+}(t)\right\rangle-\frac{\Omega}{2}\left\langle\hat{\sigma}_{z}(t)\right\rangle, \\
\frac{d}{d t}\left\langle\hat{\sigma}_{z}(t)\right\rangle=-\gamma(2 \bar{n}+1)\left\langle\hat{\sigma}_{z}(t)\right\rangle \\
+\Omega\left(\left\langle\hat{\sigma}_{-}(t)\right\rangle+\left\langle\hat{\sigma}_{+}(t)\right\rangle\right)-\gamma .
\end{array}
$$

Following the procedure outlined in Ref. [1], it is possible 
to show that

$$
\begin{aligned}
\left\langle\hat{\sigma}_{z}(t)\right\rangle & =\left(\left\langle\hat{\sigma}_{z}(0)\right\rangle+\frac{\gamma^{2}(1+2 \bar{n})}{2 \alpha \beta}\right) e^{-\beta t}-\frac{\gamma^{2}(1+2 \bar{n})}{2 \alpha \beta} \\
& +\left[\frac{\beta-\gamma(2 \bar{n}+1)}{\beta-\alpha}\left\langle\hat{\sigma}_{z}(0)\right\rangle+\frac{\gamma^{2}(1+2 \bar{n})}{2 \alpha(\beta-\alpha)}\right. \\
& \left.+\frac{\Omega}{\beta-\alpha}\left(\left\langle\hat{\sigma}_{-}(0)\right\rangle+\left\langle\hat{\sigma}_{+}(0)\right\rangle\right)-\frac{\gamma}{\beta-\alpha}\right] \\
& \times\left(e^{-\alpha t}-e^{-\beta t}\right)
\end{aligned}
$$

where

$$
\begin{aligned}
& \alpha=\frac{\gamma}{4}(6 \bar{n}+3)-\xi, \\
& \beta=\frac{\gamma}{4}(6 \bar{n}+3)+\xi,
\end{aligned}
$$

in which

$$
\xi=\left[\frac{\gamma^{2}}{16}(2 \bar{n}+1)^{2}-\Omega^{2}\right]^{1 / 2} .
$$

It may worth mentioning that in the forthcoming discussions various quantities of interest can be determined using Eq. (6).

Applying Eqs. (6), (7), (8), (9) and the fact that the population inversion, $W(t)=\left\langle\hat{\sigma}_{z}(t)\right\rangle$, it is found at steady state that

$$
W=-\frac{1}{(1+2 \bar{n})\left(\frac{2 \Omega^{2}}{\gamma^{2}}+1\right)} .
$$

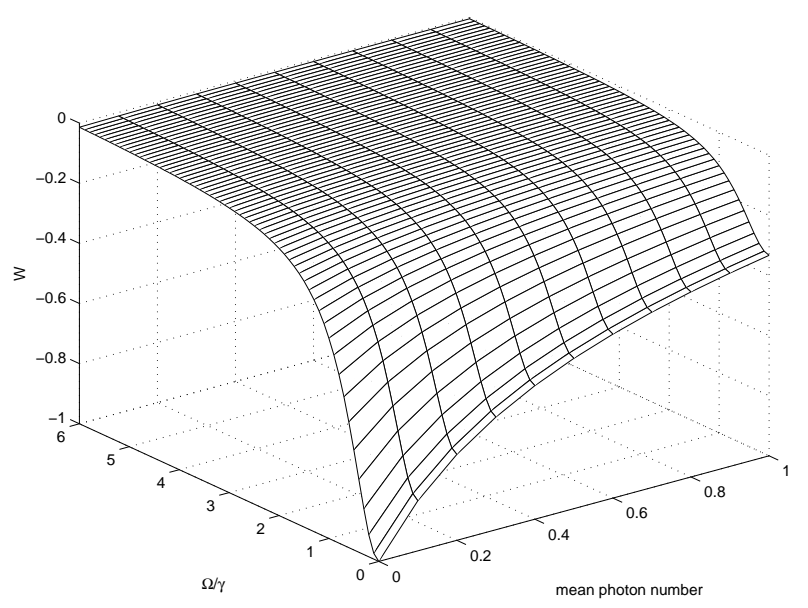

FIG. 1: Plot of the atomic inversion at steady state.

The population inversion is defined as the difference of the population in the lower and upper energy levels, $\rho_{a a}-\rho_{b b}$. From the result shown in Fig. 1, it is not difficult to observe that the population inversion increases with the amplitude of the external coherent radiation and intensity of the thermal fluctuations. Moreover, further scrutiny reveals that the inversion decreases with the intensity of the thermal fluctuations for larger values of the amplitude of the coherent radiation. As clearly shown in our previous work [1], the population inversion decreases with the increasing degree of squeeze parameter for larger values of $\Omega / \gamma$. However, comparison of the dependence of the population inversion on the intensity of the noise fluctuations in the two systems indicates that the squeezed input slightly enhances the decrement of the population inversion. Since the unbiased noise fluctuations in the thermal vibrations are presumed to be washed out in the process of calculating the mean values and hence $\bar{n}$ accounts for the intensity of the fluctuations alone. This is one of the essential differences in atomic dynamics in cases of biased or unbiased noise fluctuations are allowed to enter the cavity. It can also be inferred from this result that the atom stays more often in the lower energy level at steady state, since the population inversion is found to be negative for all values of the parameters under consideration.

Furthermore, on the basis of the fact that the probability for the atom to be in the upper energy level is given by $\rho_{a a}(t)=\frac{\left\langle\hat{\sigma}_{z}(t)\right\rangle+1}{2}$ and making use of Eq. (6) one gets at steady state

$$
\rho_{a a}=\frac{\frac{\Omega^{2}}{\gamma^{2}}+\bar{n}(1+2 \bar{n})}{\frac{2 \Omega^{2}}{\gamma^{2}}+(2 \bar{n}+1)^{2}} .
$$

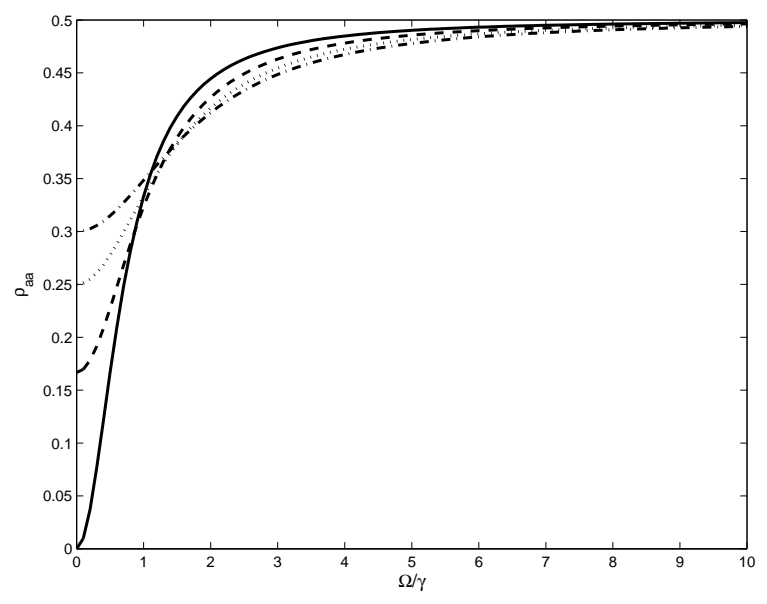

FIG. 2: Plots of the probability for the atom to be in the upper atomic energy level at steady state for $\bar{n}=0$ (solid line), $\bar{n}=0.25$ (dashed line), $\bar{n}=0.5$ (dotted line) and $\bar{n}=0.75$ (dashed-dotted line).

It can readily be seen from Fig. 2 that the probability for the atom to be in the upper energy level increases with the mean photon number of the reservoir modes for smaller values of $\Omega / \gamma$, but decreases for larger values. In relation to this, Tanas et al. 2] recently found that the absorption spectrum of the driving field or the stationary line shape, the quantity which is twice of the value 
in Eq. (11), is less than 1 at resonance when a strongly driven two-level atom is coupled to a finite band squeezed vacuum reservoir. The dependence of the probability for the atom to be in the upper energy level on the squeezed parameter has also the same form as indicated in Fig. 2. It, hence, can be inferred that when there is an external radiation the atom may absorb a photon from the cavity and then excited to the upper energy level. Even then irrespective of the strength of the external radiations (coherent driving and thermal fluctuations) the rate of emission is relatively stronger than the rate of absorption. As can readily be seen, $\rho_{a a}$ takes values between 0 and 0.5 , which implies that this mechanism perhaps be employed in preparing the atom in an arbitrary coherent superposition of the upper and lower energy levels by adjusting the required amplitude of the external coherent radiation. Despite previous claim that inhibition of absorption is related to the phase difference between the coherent and squeezed radiations, the result shown in this Section, rather, indicates that the inhibition of absorption is predominantly depends on the intensity of the fluctuations. It is good to note that there is a slight variation due to the phase sensitivity of the noise of course.

\section{EMISSION SPECTRUM}

Emission spectrum that corresponds to the power spectrum of a radiation emitted by a two-level atom can be conveniently expressed in terms of the atomic creation and annihilation operators as

$$
S(\omega)=2 \operatorname{Re} \int_{0}^{\infty}\left\langle\hat{\sigma}_{+}(t) \hat{\sigma}_{-}(t+\tau)\right\rangle_{s s} e^{i \omega \tau} d \tau
$$

Following the approach in Ref. [1] along with the aid of the properties of the atomic operators that $\left\langle\hat{\sigma}_{-}^{2}\right\rangle=0$ and $\left\langle\hat{\sigma}_{+} \hat{\sigma}_{z}\right\rangle=-\left\langle\hat{\sigma}_{+}\right\rangle$, one can obtain

$$
\begin{aligned}
& \left\langle\hat{\sigma}_{+}(t) \hat{\sigma}_{-}(t+\tau)\right\rangle=\left\langle\sigma_{+}(t) \sigma_{-}(t)\right\rangle\left\{2 e^{-\frac{\gamma}{2}(2 \bar{n}+1) \tau}\right. \\
& \left.-\frac{\Omega^{2}}{2\left(\frac{\gamma(1+2 \bar{n})}{2}-\beta\right)(\beta-\alpha)}\left(e^{-\beta \tau}-e^{-\alpha \tau}\right)\right\} \\
& +\left\langle\hat{\sigma}_{+}(t)\right\rangle\left\{\frac { - \Omega } { 2 ( \frac { \gamma ( 1 + 2 \overline { n } ) } { 2 } - \beta ) } \left[\frac{\gamma}{\beta-\alpha}-\frac{\gamma^{2}(1+2 \bar{n})}{2 \beta(\beta-\alpha)}\right.\right. \\
& \left.+\frac{\alpha-\gamma(2 \bar{n}+1)}{\beta-\alpha}\right] e^{-\beta \tau}-\frac{\Omega}{2\left(\frac{\gamma(1+2 \bar{n})}{2}-\alpha\right)}\left[\frac{\gamma^{2}(1+2 \bar{n})}{2 \alpha(\beta-\alpha)}\right. \\
& \left.-\frac{\gamma}{\beta-\alpha}+\frac{\gamma(2 \bar{n}+1)-\beta}{\beta-\alpha}\right] e^{-\alpha \tau} \\
& +\frac{\Omega \gamma^{2}}{2 \beta \alpha}\left(1-e^{\left.\left.-\frac{\gamma(1+2 \bar{n})}{2} \tau\right)\right\},}\right. \\
& \left\langle\hat{\sigma}_{+}\right\rangle_{s s}=\frac{\Omega \gamma^{2}}{2 \alpha \beta} .
\end{aligned}
$$

In order to study the dependence of the emission spectrum on the amplitude of the coherent radiation and intensity of the thermal fluctuations more closely, two cases of interest are considered. For a strong driving field, $\Omega \gg \gamma$, it is possible to easily see from Eq. (9) that $\xi=i \Omega$, as a result, $\beta-\alpha=i 2 \Omega, \frac{c \gamma}{2}-\beta=-i \Omega$, $\frac{c \gamma}{2}-\alpha=i \Omega, \alpha \beta=\Omega^{2}$, and $\left\langle\sigma_{+}(t)\right\rangle_{s s}=0$. Moreover, since $\rho_{a a}(t)=\left\langle\hat{\sigma}_{+}(t) \hat{\sigma}_{-}(t)\right\rangle$ Eq. (11) reduces for $\Omega \gg \gamma$ and modest values of $\bar{n}$ to

$$
\left\langle\sigma_{+}(t) \sigma_{-}(t)\right\rangle_{s s}=\frac{1}{2} .
$$

It can be realized that, at steady state, the population is independent of the strength of the decoherence which is consistent with the result shown in Fig. 2. This would strengthen the already established fact that to prepare a two-level atom in a possible maximum coherent superposition of the two energy levels, driving it with a strong external coherent radiation is sufficient. Furthermore, it is not difficult to see that

$$
\begin{aligned}
\left\langle\hat{\sigma}_{+}(t) \hat{\sigma}_{-}(t+\tau)\right\rangle_{s s} & =\frac{1}{4} e^{-\frac{\gamma}{2}(2 \bar{n}+1) \tau} \\
& +\frac{1}{8} e^{\left(i \Omega-\frac{\gamma}{4}(6 \bar{n}+3)\right) \tau} \\
& +\frac{1}{8} e^{-\left(\frac{\gamma}{4}(6 \bar{n}+3)+i \Omega\right) \tau},
\end{aligned}
$$

from which follows

$$
\begin{aligned}
S(\omega) & =\frac{\frac{\gamma}{16}(6 \bar{n}+3)}{(\Omega+\omega)^{2}+\left[\frac{\gamma}{4}(6 \bar{n}+3)\right]^{2}} \\
& +\frac{\frac{\gamma}{16}(6 \bar{n}+3)}{(\Omega-\omega)^{2}+\left[\frac{\gamma}{4}(6 \bar{n}+3)\right]^{2}} \\
& +\frac{\frac{\gamma}{4}(1+2 \bar{n})}{\omega^{2}+\left[\frac{\gamma}{2}(1+2 \bar{n})\right]^{2}} .
\end{aligned}
$$

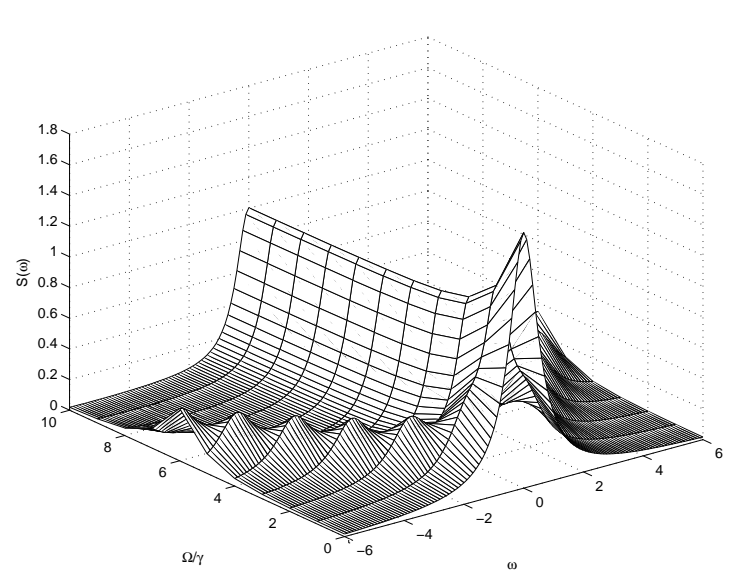

FIG. 3: Plot of the emission spectrum at steady state for $\bar{n}=0.5$. 


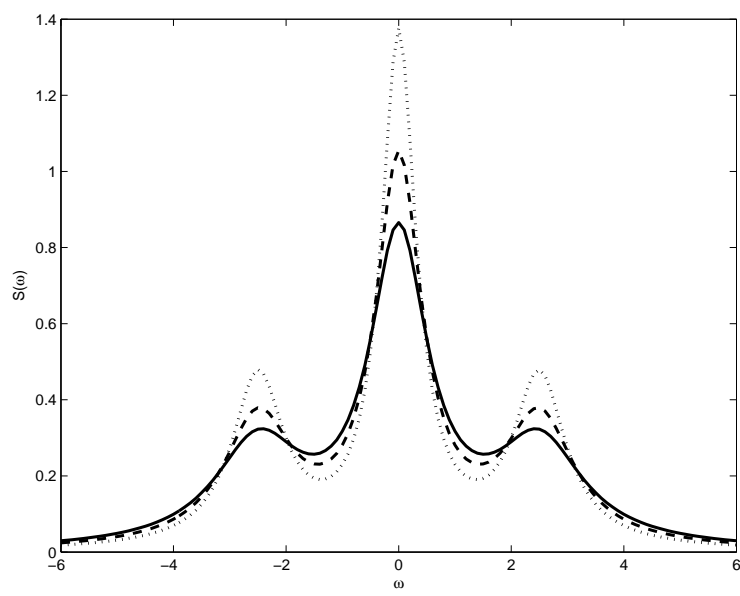

FIG. 4: Plots of the emission spectrum at steady state for $\Omega=2.5 \gamma, \bar{n}=0.75$ (solid line), $\bar{n}=0.5$ (dashed line) and $\bar{n}=0.25$ (dotted line).

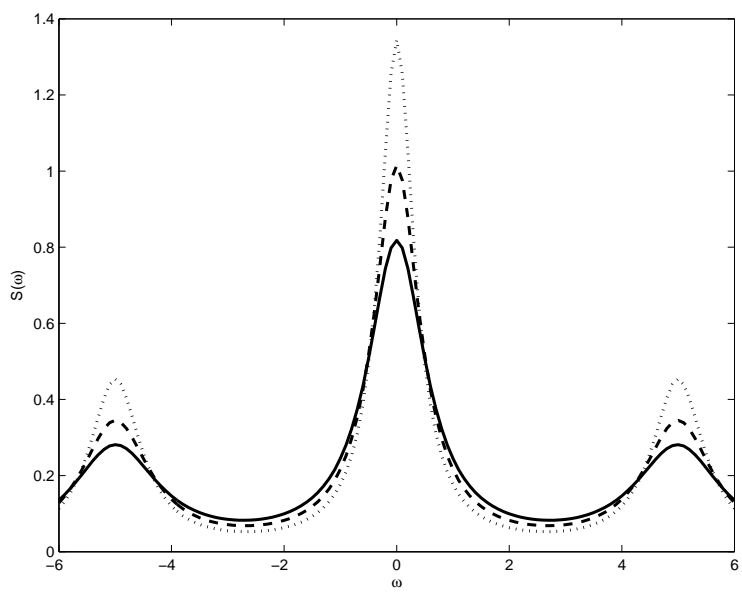

FIG. 5: Plots of the emission spectrum at steady state for $\Omega=5 \gamma, \bar{n}=0.75$ (solid line), $\bar{n}=0.5$ (dashed line) and $\bar{n}=0.25$ (dotted line).

It is not difficult to observe that the emission spectrum has three well defined peaks at $\omega=0$ and $\omega= \pm \Omega$ with line width of $\frac{\gamma(1+2 \bar{n})}{2}$ and $\frac{\gamma(6 \bar{n}+3)}{4}$. For $\bar{n}=0$, this result goes over to the usual Mollow type resonant fluorescent spectrum [9]. As can easily be seen from Fig. 3, the stronger the intensity of the thermal fluctuations of the noise, the wider the splitting and the shorter the height of the spectrum would be. In addition, comparison of the results given in Figs. 4 and 5 shows that the width of the central line and sidebands broadened with the intensity of the decoherence, whereas the heights decreased. In connection to this, Parkins [11] has simulated the resonance fluorescence of a two-level atom coupled to a twomode squeezed vacuum reservoir and found that all the three peaks exhibit subnatural line widths for particular choice of the phase in a strong driving limit for a mod- erate squeezed input. On the other hand, Tanas et al. 22 recently found that the spectral lines of the resonance fluorescence of the two-level atom coupled to finite band squeezed vacuum reservoir are narrower than for the ordinary vacuum and the side bands are slightly shifted. It is now evident that the profile of the spectra are the same as what is obtained here even when the biased noise is replaced by unbiased noise fluctuations. Nonetheless, comparison with previous results shows that the biased fluctuations in the squeezed vacuum modes suppress the height of the central peak prominently than the unbiased noise fluctuations in the decoherence phenomenon, which is basically related to the phase sensitivity in the squeezed input. On the basis of this understanding, one can then come to conclude that except for such minor differences, the essential mechanism in emission-absorption process depends on the intensity of the fluctuations of the noise associated with the environment rather than the phase.

In a weak driving limit, $\Omega \approx 0$, it follows from Eq. (13) that

$$
\left\langle\hat{\sigma}_{+}(t) \hat{\sigma}_{-}(t+\tau)\right\rangle_{s s}=2\left\langle\hat{\sigma}_{+}(t) \hat{\sigma}_{-}(t)\right\rangle_{s s}\left[e^{-\frac{\gamma}{2}(2 \bar{n}+1) \tau}\right]
$$

which leads, making use of Eq. (11) at steady state, $\left\langle\hat{\sigma}_{+}(t) \hat{\sigma}_{-}(t)\right\rangle_{s s}=\frac{\bar{n}}{2 \bar{n}+1}$, to

$$
\left\langle\hat{\sigma}_{+}(t) \hat{\sigma}_{-}(t+\tau)\right\rangle_{s s}=\frac{\overline{2 n}}{2 \bar{n}+1}\left[e^{-\frac{\gamma}{2}(2 \bar{n}+1) \tau}\right] .
$$

It is, hence, observed that in the weak driving limit the noise associated to the thermal fluctuations can excite the atom to the upper energy level, namely, for strongly intense thermal light there is nearly $50 \%$ probability for the atom to be found in the upper atomic energy level at steady state. Just like the coherent driving radiation, the thermal fluctuations entering the cavity through the walls of the mirror can also be employed in preparing the atom in arbitrary coherent superposition of the two atomic energy levels. One can easily see that the atom would be completely in the ground state at steady state for vacuum reservoir. Moreover, it can be deduced from Eq. (19) that for a weak driving limit, $\Omega=0$, the emission spectrum generally does not split. Therefore, it is possible to infer that the spectral splitting is associated with the strength of the external coherent radiation, whereas broadening of the width with the intensity of the fluctuations entering the cavity.

\section{PHOTON STATISTICS OF THE CAVITY RADIATION}

Currently available literatures indicate that the photon statistics of the cavity radiation can be investigated using the normalized two-time second-order correlation function that can be expressed for the two-level atom in 
terms of the creation and annihilation atomic operators in the form

$$
g^{(2)}(\tau)=\frac{\left\langle\hat{\sigma}_{+}(t) \hat{\sigma}_{+}(t+\tau) \hat{\sigma}_{-}(t+\tau) \hat{\sigma}_{-}(t)\right\rangle}{\left\langle\hat{\sigma}_{+}(t) \hat{\sigma}_{-}(t)\right\rangle^{2}} .
$$

Therefore, in view of the property of the atomic operators,

$$
\left\langle\hat{\sigma}_{+}(t+\tau) \hat{\sigma}_{-}(t+\tau)\right\rangle=\frac{\left\langle\hat{\sigma}_{z}(t+\tau)\right\rangle+1}{2},
$$

one gets

$$
g^{(2)}(\tau)=\frac{\left\langle\hat{\sigma}_{+}(t) \hat{\sigma}_{-}(t)\right\rangle+\left\langle\hat{\sigma}_{+}(t) \hat{\sigma}_{z}(t+\tau) \hat{\sigma}_{-}(t)\right\rangle}{2\left\langle\hat{\sigma}_{+}(t) \hat{\sigma}_{-}(t)\right\rangle^{2}},
$$

from which follows

$$
\begin{aligned}
g^{(2)}(\tau) & =\frac{1}{2\left\langle\hat{\sigma}_{+}(t) \hat{\sigma}_{-}(t)\right\rangle}\left[\frac{2 \alpha \beta-\gamma^{2}(1+2 \bar{n})}{2 \alpha \beta}\right. \\
& +\frac{2 \beta \alpha-\gamma^{2}(1+2 \bar{n})-4 \beta \gamma \bar{n}}{2 \beta(\beta-\alpha)} e^{-\beta \tau} \\
& \left.+\frac{\gamma^{2}(1+2 \bar{n})-2 \beta \alpha+4 \alpha \gamma \bar{n}}{2 \alpha(\beta-\alpha)} e^{-\alpha \tau}\right] .
\end{aligned}
$$

For a strong driving limit one finds at steady state

$$
g^{(2)}(\tau)=1-\cos (\Omega \tau) e^{-\frac{\gamma}{4}(6 \bar{n}+3) \tau} .
$$

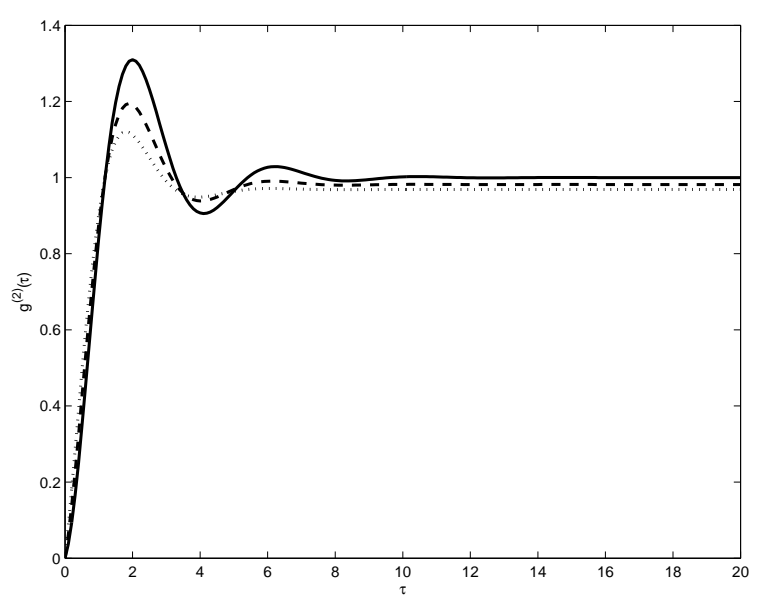

FIG. 6: Plots of the two-time second-order correlation function (Eq. (23)) at steady state for $\Omega=3 \gamma, \bar{n}=0.75$ (solid line), $\bar{n}=0.5$ (dashed line) and $\bar{n}=0.25$ (dotted line).

It is known for long that the two-time second-order correlation function describes the delayed coincidence between the successively emitted light. It is not difficult to see from Figs. 6, 7 and 8 that $g^{(2)}(\tau)>g^{(2)}(0)$ for all

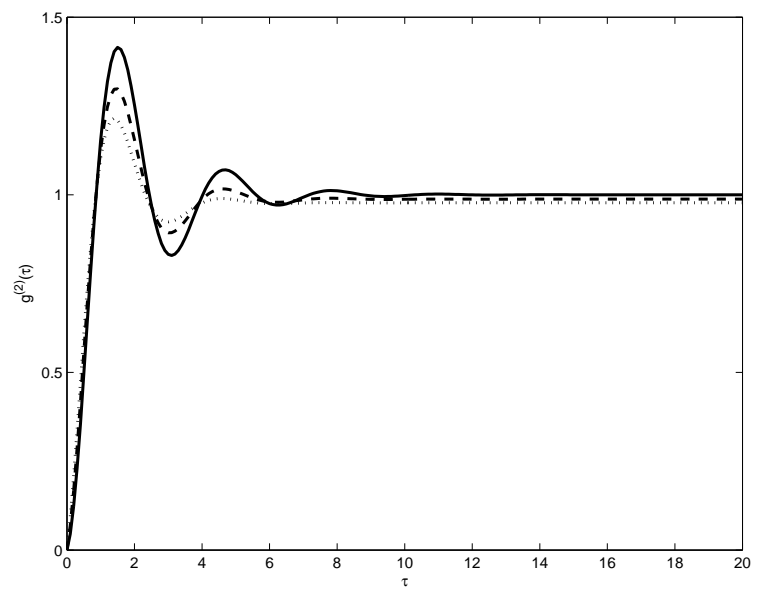

FIG. 7: Plots of the two-time second-order correlation function (Eq. (23)) at steady state for $\Omega=4 \gamma, \bar{n}=0.75$ (solid line), $\bar{n}=0.5$ (dashed line) and $\bar{n}=0.25$ (dotted line).

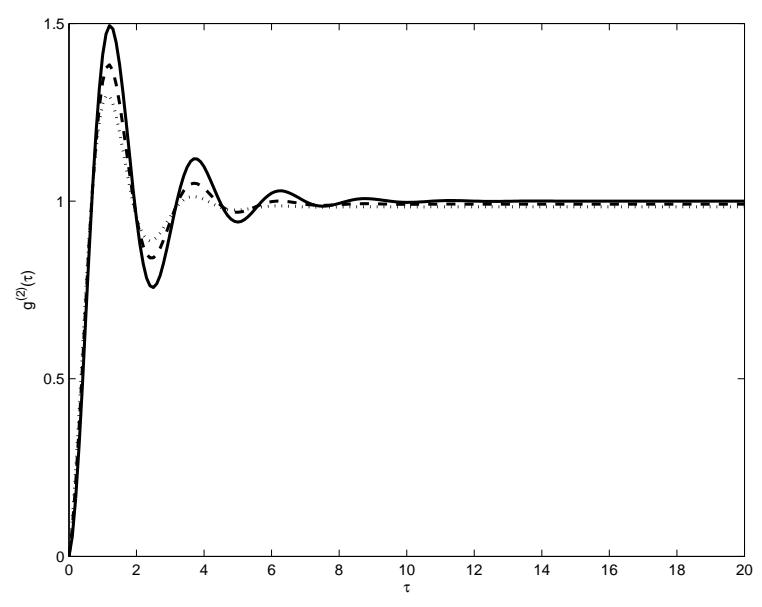

FIG. 8: Plots of the two-time second-order correlation function (Eq. (23)) at steady state for $\Omega=5 \gamma, \bar{n}=0.75$ (solid line), $\bar{n}=0.5$ (dashed line) and $\bar{n}=0.25$ (dotted line).

cases under consideration, which indicates that the emitted light exhibits photon anti-bunching, despite the fact that the unbiased noise fluctuations entering the cavity destroys the quantum features of the radiation. The antibunching phenomenon can be interpreted as the atom goes over to the lower energy level after emitting a photon needs time before it absorbs a photon and excited to the upper energy level to emit the next photon. It can be deduced that this is one of the fundamental properties of the absorption and emission processes of the two-level atom, which is independent of the external coherent radiation and reservoir to which the cavity is coupled. It is also possible to identify the photon statistics of the cavity radiation employing the normalized two-time secondorder correlation function. As can readily be seen from Figs. 6, 7 and 8, the two-time second-order correlation function oscillates between $g^{(2)}(\tau)>1$ and $g^{(2)}(\tau)<1$ for smaller values of the delayed time. This can be in- 
terpreted as the photon statistics oscillates between sub and supper-Poissonian in this case. However, for larger delayed time there is a possibility that $g^{(2)}(\tau)>1$ or $g^{(2)}(\tau)<1$ depending on the amplitude of the coherent radiation and the strength of the decoherence. The super-Poissonian photon statistics becomes more prominent for stronger intensity of decoherence. As can be observed from Eq. (24) the emitted photon exhibits Poissonian photon statistics for modest values of the amplitude of the external radiation and for larger delayed time. Similar oscillatory nature of the two-time second-order correlation function with delayed time has been discussed by various authors [1, 10]. In particular, D'Souza et al. [10] earlier predicted that the emitted radiation exhibits both sub and super-Poissonian photon statistics based on the phase between the coherent and squeezed lights from the curve of the Mandel's response function, whereas recent study shows that for larger delayed time the cavity radiation exhibits a super-Poissonian photon statistics when the cavity is coupled to a broadband squeezed vacuum reservoir [1]. It is evident from these works that the photon statistics at larger delayed time is dominated by the properties of the light entering the cavity.

\section{SQUEEZING OF THE CAVITY RADIATION}

The squeezing properties of the cavity radiation can be studied applying the variances of the atomic-dipole operators in the normal order. In order to determine the variances of the atomic-dipole operators in the normal order, it is possible to begin with a well established fact that the emitted radiation can be described in terms of the electric field. If the two quadrature components of the electric field satisfy the commutation relation,

$$
\left[\hat{E}_{\theta}, \hat{E}_{\theta-\pi / 2}\right]=i 2 C
$$

then the usual uncertainty relation,

$$
\left\langle\left(\Delta \hat{E}_{\theta}\right)^{2}\right\rangle\left\langle\left(\Delta \hat{E}_{\theta-\pi / 2}\right)^{2}\right\rangle \geq C^{2}
$$

holds. The radiation represented by this electric field is in squeezed state, provided that either $\left\langle\left(\Delta \hat{E}_{\theta}\right)^{2}\right\rangle$ or $\left\langle\left(\Delta \hat{E}_{\theta-\pi / 2}\right)^{2}\right\rangle$ is below the vacuum limit $C$. In general, one of the variances of the electric field can be put in the normal order as

$$
\left\langle:\left(\Delta \hat{E}_{\theta}\right)^{2}:\right\rangle=\left\langle\left(\Delta \hat{E}_{\theta}\right)^{2}\right\rangle-C,
$$

where the symbol :: stands for the operator put in the normal order. Therefore, the squeezing can be related to the requirement that either $\left\langle:\left(\Delta \hat{E}_{\theta}\right)^{2}:\right\rangle$ or $\left\langle:\left(\Delta \hat{E}_{\theta-\pi / 2}\right)^{2}:\right\rangle$ is less than zero. On the other hand, making use of the relation between the electric field and atomic operators, the variance in the field operator can be defined in terms of the atomic-dipole operators. In this regard, Ficek and Tanas [23] have expressed the variance of the atomic-dipole operator in the normal order in the form

$$
\left\langle:\left(\Delta \hat{\sigma}_{i}\right)^{2}:\right\rangle=\left\langle\left(\Delta \hat{\sigma}_{i}\right)^{2}\right\rangle+\frac{\left\langle\hat{\sigma}_{z}\right\rangle}{2}
$$

where $i=x, y$ and

$$
\begin{aligned}
& \hat{\sigma}_{x}=\frac{1}{\sqrt{2}}\left(\hat{\sigma}_{+}+\hat{\sigma}_{-}\right), \\
& \hat{\sigma}_{y}=\frac{i}{\sqrt{2}}\left(\hat{\sigma}_{-}-\hat{\sigma}_{+}\right) .
\end{aligned}
$$

Then with the aid of the fact that at steady state $\left\langle\hat{\sigma}_{-}(t)\right\rangle_{s s}=\left\langle\hat{\sigma}_{+}(t)\right\rangle_{s s}$, one finds

$$
\begin{gathered}
\left\langle:\left(\Delta \hat{\sigma}_{x}\right)^{2}:\right\rangle=\frac{1}{2}\left(1+\left\langle\hat{\sigma}_{z}\right\rangle\right)-2\left\langle\hat{\sigma}_{+}\right\rangle_{s s}^{2}, \\
\left\langle:\left(\Delta \hat{\sigma}_{y}\right)^{2}:\right\rangle=\frac{1}{2}\left(1+\left\langle\hat{\sigma}_{z}\right\rangle\right) .
\end{gathered}
$$

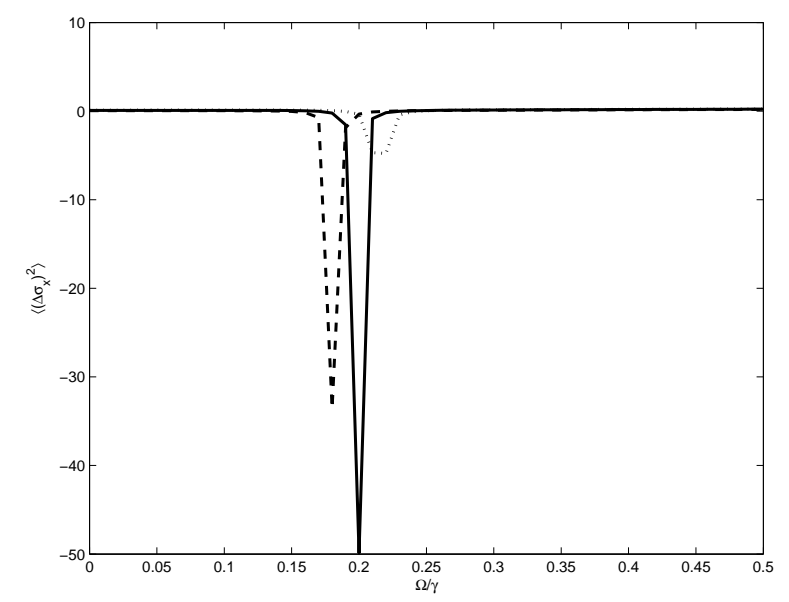

FIG. 9: Plots of the squeezing of the cavity radiation at steady state for $\bar{n}=0.05$ (dotted line), $\bar{n}=0.1$ (solid line) and $\bar{n}=0.15$ (dashed line).

It is not difficult to realize based on the definition of the population inversion along with the result shown in Fig. 1 that $\left\langle\Delta \hat{\sigma}_{z}\right\rangle>-1$, which implies that $\left\langle:\left(\Delta \hat{\sigma}_{y}\right)^{2}:\right\rangle$ never be negative at steady state. As can also be seen from Eq. (14), $\left\langle\hat{\sigma}_{+}\right\rangle_{s s}=\gamma^{2} / 2 \Omega$, which approaches zero for strong driving radiation. In this case as well, it can readily be seen that $\left\langle:\left(\Delta \hat{\sigma}_{x}\right)^{2}:\right\rangle$ never be negative. This indicates that the emitted radiation does not exhibit squeezing when the atom is pumped externally with a strong coherent radiation at steady state. As opposed to this, $\left\langle:\left(\Delta \hat{\sigma}_{x}\right)^{2}:\right\rangle$ can be less than zero for certain values of the amplitude of the external radiation as clearly shown in Fig. 9 for smaller values of $\Omega / \gamma$. On the basis of the criterion set for squeezing, the light emitted by the 
two-level atom exhibits squeezing even in the presence of a significant amount of decoherence that is believed to destroy the quantum features of the light. Unfortunately, the squeezing is found to exist only for narrow pockets of the values of the amplitude of the coherent radiation which, of course, depend on the strength of the decoherence. This result demonstrates that the atomic coherence induced between the upper and lower energy levels by the coherent radiation, which is responsible for the squeezing, is too strong to be destroyed by the unbiased noise fluctuations. It is believed that this must be the reason for observing a considerable entanglement in a correlated emission laser even in the presence of a strong decoherence [24]. Moreover, critical survey of Fig. 9 reveals that the squeezing exists for values of $\Omega / \gamma$ for which the squeezing disappears in the absence of the decoherence. This may be related to a recent claim that a decoherence due to environment enhances entanglement in a two-level atomic system by providing an indirect correlation between totally uncorrelated quantum states [25].

\section{CONCLUSION}

In this contribution, a thorough study of the effects of the external coherent radiation and thermal fluctuations corresponding to the vibration of the walls of the mirrors due to their coupling with the external environment on the atomic dynamics, squeezing properties and photon statistics of the radiation produced by a coherently driven two-level atom trapped in a resonant cavity is presented. It is found that though the atom absorbs the radiation from the available cavity modes, including the driving, emitted and thermal light entering the cavity, and makes a transition to the upper energy level, it prefers to stay in the ground state more often irrespective of the amplitude of the coherent radiation and the strength of the intensity of the decoherence. In this regard, in comparison to previous works, the fundamental phenomenon in absorption and emission processes, in which the rate of emission is greater than the rate of ab- sorption, is basically found to depend on the strength of the fluctuations associated to the environment rather the phase difference. This, on the other hand, indicates that except for the minor differences in its degree the inhibition of absorption is resulted when the cavity is coupled to both biased and unbiased noise fluctuations. Therefore, it is possible to deduce from this study that predominantly the atomic dynamics is affected by the mean photon number of the reservoir modes rather than whether the reservoir is squeezed vacuum or thermal. In addition to this, it is not difficult to realize that the twolevel atom can be prepared in arbitrary coherent superposition of the upper and lower energy levels by varying the intensity of the thermal fluctuations in the environment. It is believed that this approach perhaps would be practically attractive in the preparation of the injected atomic coherence required in multi-level atomic laser [24].

It was previously discussed that the emission spectrum is broadened and the height is reduced by the squeezed input. In the same way, the thermal fluctuation is found to broaden the spectrum and reduce the height significantly, but it does not contribute to the splitting of the central line into triplet. Comparison with the previous works indicates that the biased noise fluctuations in the squeezed vacuum modes decrease the height of the central peak more than the unbiased noise fluctuations in decoherence. Moreover, the emitted radiation is found to exhibit anti-bunching photon statistics independent of the type of the reservoir. It is, rather, a fundamental property of a driven two-level atom related to the time required for absorbing a radiation to make a transition to the upper energy level after the atom emits a photon. As opposed to this, the super-Poissonian statistics is found to be enhanced by biased noise fluctuations. In addition to this, the cavity radiation exhibits appreciable squeezing for some pockets of the amplitude of the driving radiation that depends on the strength of the intensity of decoherence. In conclusion, even though the successively emitted photons are separated in time, they are strongly correlated which leads to the appearance of the nonclassical features even in the presence of decoherence which presumably destroys the quantum properties.
[1] S. Tesfa, J. Mod. Opt. 541759 (2007).

[2] R. Tanas, Z. Ficek, A. Messikh and T. El-Shahat, J. Mod. Opt. 451859 (1998).

[3] C. W. Gardiner, Phys. Rev. Lett. 561917 (1986).

[4] H. J. Carmichael, A. S. Lane and D. F. Walls, J. Mod. Opt. 34821 (1987).

[5] J. M. Courty and S. Reynaud, Europhys. Lett. 10237 (1989).

[6] R. Tanas, J. Opt. B: Quantum Semiclass. Opt. 4142 (2002).

[7] A. Kowalewska-Kudlaszyk and R. Tanas, J. Mod. Opt. 48347 (2001).

[8] B. J. Dalton, Z. Ficek and S. Swain, J. Mod. Opt. 46379
(1999).

[9] B. R. Mollow, Phys. Rev. 1881969 (1969).

[10] R. D'Souza, A. S. Jayaroa and S. V. Lawande, Phys. Rev. A 414083 (1990).

[11] A. S. Parkins, Phys. Rev. A 426873 (1990).

[12] A. S. Parkins and C. W. Gardiner, Phys. Rev. A 373867 (1988).

[13] H. J. Carmichael, A. S. Lane and D. F. Walls, Phys. Rev. Lett. 582539 (1987).

[14] C. H. Keitel, P. L. Knight, L. M. Narducci and M. O. Scully, Opt. Commun. 118143 (1995).

[15] C. H. Keitel, J. Mod. Opt. 431555 (1996).

[16] M. Bosticky, Z. Ficek and B. J. Dalton, Phys. Rev. A 53 
4439 (1996).

[17] P. R. Rice and C. A. Baird, Phys. Rev. A 533633 (1996).

[18] W. S. Smyth and S. Swain, Phys. Rev. A 532846 (1996).

[19] S. K. Lee and H. Lee, Phys. Rev. A 74063817 (2006).

[20] G. J. Milburn, Phys. Rev. A 445401 (1991).

[21] P. R. Puri and G. S. Agarwal, Phys. Rev. A 353433 (1996).
[22] W. H. Louisell, Quantum statistical properties of radiation (Wiley, Newyork, 1973).

[23] Z. Ficek and R. Tanas, Physics Reports 372369 (2002).

[24] S. Tesfa, J. Phys. B: At. Mol. Opt. Phys. 402373 (2007).

[25] J. Zhang and H. Yu, Phys. Rev. A 75012101 (2007). 